(From the Laboratory of Microbiology, Technical University, Delft).

\title{
SOME OBSERVATIONS ON THE METABOLISM OF BACTERIA OXIDIZING MOLECULAR HYDROGEN
}

\author{
by
}

\section{A. J. KLUYVER and A. MANTEN}

(Received June 16, 1942).

\section{BRIEF REVIEW OF PREVIOUS INVESTIGATIONS ON HYDROGEN OXIDIZING BACTERIA.}

Already in 1839 DE SAUSSURE (1) observed that in the presence of soil a mixture of hydrogen and oxygen gradually disappeared and apparently was converted into water. In 1892 IMMENDORF (2) reinvestigated this phenomenon and observed along with other facts that addition of chloroform to the soil prevented the gas consumption. In this way the biological nature of the process had been made extremely probable.

Experimental proof that bacteria were responsible for the hydrogen oxidation was brought almost simultaneously by NIKLEWSKI (3) and by KASERER (4). The latter inoculated a purely mineral medium with scme garden soil and found that on bringing this medium into contact with a gas mixture containing hydrogen, axygen and carbon dioxide a bacterial film developed which actively oxidized the hydrogen. KASERER gave a description of the organism to which he gave the name of Bacillus pantotrophus. He failed, however, in his attempts to bring it into pure culture. NIKLEwSKI made more or less similar observations.

LEBEDEFF (5) was the first in claiming to have obtained a pure culture of a hydrogen oxidizing bacterium, but he does not give many particulars regarding the organism, nor regarding the procedure applied in its isolation. He made, however, many experiments regarding the ratio of the amounts of hydrogen and of oxygen consumed under different conditions. This ratio proved to be subject to rather considerable fluctuations. Since, however, LEBEDEFF also established that the nitrate present in his medium was reduced and was partly converted into elementary nitrogen, it seems likely that the evolution of this gas will have impaired in some measure the correctness of the analyses made. 
In a continuation of his earlier studies NIKLEWSKI (6) reported the isolation of two different hydrogen oxidizing bacteria which he brought into the genus Hydrogenomonas, previously created by ORLA-JENSEN. NIKLEWSKr found that neither his Hydrogenomonas vitrea, nor his Hydrogenomonas flava were able to grow for themselves in the hydrogen-oxygen atmosphere used in the enrichment culture. This situation changed, however, when the oxygen tension in the atmosphere was lowered to $53 \mathrm{~mm}$ mercury. He also established the heterotrophic mode of -life of both species by showing that they were able to grow in the absence of hydrogen if suitable organic substrates were made available. Such substrates prevented more or less completely the oxidation of hydrogen. In consequence hereof he emphasizes the importance of a further study of the mechanism of this oxidation for the obtaining of a better insight into respiration processes in general.

The results of a very thorough investigation of bacterial hydrogen oxidation have been published by RuHLand (7) in 1924 .

RUHLAND made a great number of experiments regarding the gaseous metabolism of growing cultures of Bacillus pycnoticus, a spore-forming rod which actively oxidized hydrogen. In the first place he could corroborate NIKLEWSKI's observation that addition of organic substrates, like glucose and mannitol, to the culture medium inhibits the oxidation of hydrogen. RuHLAND then very carefully determined the ratio between. the amounts of hydrogen and oxygen consumed, and established that this ratio as a rule surpasses the theoretical value 2 quite considerably. Values between 2.78 and 2.14 were found. Moreover, RuHLAND could prove that the surplus of hydrogen consumed corresponded quite satisfactorily with the amount of carbon dioxide assimilated in a proportion of $2: 1$, which fact strongly indicates the carbohydrate nature of the assimilation product formed. In a few experiments in which the hydrogen oxidizing capacity of pre-formed bacteria were tested in the absence of carbon dioxide the hydrogen/oxygen ratio was determined as 1.80 . Here apparently the assimilation process was practically excluded, whilst part of the oxygen present was used in the oxidation of cell material. We will postpone the discussion of the general conclusions drawn by RUHLAND from his experiments to the next chapter.

At about the same time a paper was published by RuHLAND's collaborator GrohmanN (8), in which paper the wide distribution of bacteria capable of hydrogen oxidation in nature was amply demonstrated. GroHMANN showed that this faculty is encountered amongst representatives of widely different natural groups of bacteria. This point of view 
has since been confirmed by several other workers and forms a strong argument against the still prevailing tendency of uniting all hydrogen oxidizing bacteria in a separate genus: Hydrogenomonas.

Mention should be made also of GROHMANN's remarkable experience that after his removal from Tübingen to Leipzig all his cultures spontaneously lost their ability to bring about hydrogen oxidation, and of the even more startling fact that new attempts to obtain enrichment cultures remained unsuccessfull.

In a recent publication of LEE and UMBREIT (9) experiments ale described in which crude sand cultures showed an active consumption of hydrogen as long as oxygen was also present in the gas mixture; in contrast herewith no hydrogen was consumed when oxygen was absent, although carbon dioxide was amply available. On the other hand the authors conclude from their experimental data that carbon dioxide is essential for the initiation of the hydrogen oxidizing process. From the crude cultures several pure cultures were isolated, two of which were subjected to a closer study. For these strains both autotrophic and heterotrophic growth could be proved.

\section{OUtLine OF the PRESENT INVESTIGATION.}

The studies referred to in Chapter 1 - especially those made by KASERER, NIKLEWSKI and RUHIAND - leave no doubt that at least several hydrogen oxidizing bacteria have the ability of autotrophic growth, i.e., that they are able to proliferate in a medium in which carbon dioxide is the only available carbon compound.

Now it has to be acknowledged that the way in which chemo-autotrophic organisms manage to convert carbon dioxide into cell material is still completely wrapped in mystery. As a rule comment is restricted to the remark that the energy derived from the oxidation of the inorganic substrate is amply sufficient to meet the energy requirements involved in the reduction of the carbon dioxide. However, for the better-known autotrophic bacteria, like the sulphur bacteria and the nitrifying bacteria, scarcely any attempt has been made to specify the chemical reactions which lead from carbon dioxide to cell material. Yet in the case of the last-mentioned autotrophonts it seems certain that the oxidation of the inorganic substrates proceeds in stages, involving the formation of several intermediate products. For this reason it remains quite conceivable - and it has in fact been suggested - that it is a reaction between one of these intermediates and the carbon dioxide which leads to a primary reduction of this molecule. 
In the case of the hydrogen oxidizing bacteria this ,intermediate hypothesis", however, can at once practically ${ }^{1}$ ) be ruled out, the energy yielding reaction being restricted to the simple equation:

$$
2 \mathrm{H}_{2}+\mathrm{O}_{2} \rightarrow 2 \mathrm{H}_{2} \mathrm{O} \text {. }
$$

For this reason the hydrogen oxidizing bacteria seem to offer an exceptionally favourable subject for a closer study of the chemistry of chemo-autotrophy.

In the light of this introduction it seems desirable to report here briefly the views regarding this point as laid down in the publications reviewed in Chapter 1.

The earlier investigators like KASERER and NIKLEWSKI were inclined to accept the idea that the primary reaction is one between hydrogen and carbon dioxide leading to the formation of some product of carbohydrate nature and that the oxygen only reacts with this organic substrate in an ordinary respiration process.

Thus for instance KASERER advances the following equations for the metabolic changes involved:

$$
\begin{aligned}
& \text { I. } \mathrm{H}_{2} \mathrm{CO}_{3}+2 \mathrm{H}_{2} \rightarrow \mathrm{CH}_{2} \mathrm{O}+2 \mathrm{H}_{2} \mathrm{O}+6 \mathrm{cal} \text {. } \\
& \text { II. } \mathrm{CH}_{2} \mathrm{O}+\mathrm{O}_{2} \rightarrow \mathrm{H}_{2} \mathrm{CO}_{3}+132 \mathrm{cal} .
\end{aligned}
$$

As will be seen KASERER rejects the idea of a direct reaction between hydrogen and oxygen. It must, however, be remarked that his scheme bears a fully hypothetical character, since KASERER does not offer any experimental proof that hydrogen consumption also occurs in the absence of oxygen.

As a result of his extensive investigations RUHLAND has arrived at a very different view which agrees fairly closely with that put forward by LEBEDEFF in 1909. RUHLAND concludes from his experiments that Bacillus pycnoticus is indeed able to oxidize hydrogen according to the equation:

$$
\text { I. } \mathrm{H}_{2}+1 / 2 \mathrm{O}_{2} \rightarrow \mathrm{H}_{2} \mathrm{O}+69 \mathrm{cal} \text {. }
$$

As for the carbon dioxide assimilation a plant physiologist like RuHLAND could in 1924 only visualize a reaction between carbon dioxide and water as is realized in the photo-autotrophic green cells, and he thus accepts here as well the following reaction to take place:

1) The intermediate formation of hydrogen peroxide cannot be excluded, but this does not seem to be helpful for the solution of the problem under discussion. 


$$
\text { II. } \mathrm{CO}_{2}+\mathrm{H}_{2} \mathrm{O} \rightarrow \mathrm{CH}_{2} \mathrm{O}+\mathrm{O}_{2}-112 \mathrm{cal} \text {. }
$$

The assumption, therefore, is made that in some way or other an energy transfer between the reactions I and II occurs, the average efficiency of which was calculated by RUHLAND as to be about $20.5 \%$.

According to RuHLAND the oxygen evolved in the assimilatory process would be responsible for the fact that in his growing cultures the ratio between the hydrogen and oxygen disappearing from the gas phase was always markedly higher than 2 .

The observation that in the experiments in which carbon dioxide was absent the said ratio was distinctly lower than 2 makes, however, KuHIAND conclude that the bacteria in question also bring about a normal respiration process:

$$
\text { III. } \mathrm{CH}_{2} \mathrm{O}+\mathrm{O}_{2} \rightarrow \mathrm{CO}_{2}+\mathrm{H}_{2} \mathrm{O}+112 \mathrm{cal} \text {. }
$$

The fact that hydrogen oxidizing bacteria are also able to lead a heterotrophic mode of life leaves no doubt that under these conditions such an oxidation does indeed occur. RUHLAND, however, postulates that this normal respiration with the primary assimilation product as a substrate also takes place under conditions of autotrophy, although in this case the simultaneous carbon dioxide assimilation obscures its presence:

In the light of our present knowledge RUHLAND's theory - which has the advantage that it fully corresponds with the current conceptions regarding chemo-autotrophy in other bacterial groups - presents various weak points. It seems quite improbable that the facultatively heterotrophic hydrogen oxidizing bacteria will be able to liberate oxygen from water. In this respect it must be observed that nowadays it is generally accepted that in carbon dioxide assimilation also other hydrogen donators than water may be active, so that there is no necessity whatever to accept RuHLAND's equation II. Moreover the recent developments in our insight into the chemistry of respiration seem to make it superfluous and even illogical to bring about a clear cut separation between the energy yielding reaction I and the normal respiratory process III. Since we have learnt to see the initial step of respiration as a dehydrogenation of the substrate, it appears more probable that the dehydrogenase active in this step will also be responsible for the activation of the atoms in the gaseous hydrogen. In this way the reported inhibitory effect of organic substrates on the hydrogen oxidation can be easily accounted for. The fact that the ability of hydrogen oxidation is not restricted to a small group of specialists, but is so widely distributed 
A. J. Kluyver and A. Manten,

amongst representatives of very different heterotrophic groups speaks also in favour of this view.

Mention may be made of the fact that Burk (10) in a purely theoretical study has also rejected the conclusions drawn by RUHLAND from his experiments, and BuRK again advances the view of the older investigators, namely that all the hydrogen consumed is used to reduce carbon dioxide, and all the oxygen consumed is therefore used in respiration. No direct reaction between oxygen and hydrogen would take place.

In the recent publication of LEE and UMBREIT (9) this controversy is not discussed, but it is more or less incidentally stated that the crude sand cultures used in their experiments did not consume hydrogen in the presence of carbon dioxide, if oxygen was absent, a result which seems incompatible with KASERER's scheme.

The foregoing may suffice to warrant the conclusion that the whole problem of the metabolism of hydrogen oxidizing bacteria is still badly in want of further elucidation.

In considering the situation we were struck by the fact that practically all the earlier investigations on hydrogen oxidation had been dealing with experiments of long duration, i.e., with experiments in which the growth of the bacteria was an essential factor. It appeared to us that possibly some progress could be made by. studying the behaviour of so-called , resting bacteria" in experiments of short duration, thus avoiding the complications involved in the growth process.

More especially the manometric method seemed well adapted to obtain a better insight into the interrelationship between hydrogen oxidation and normal respiration. In addition this method seemed suitable to give a definite answer to the question whether a reaction between hydrogen and carbon dioxide does occur, if oxygen is absent.

\section{ISOLATION OF PURE CULTURES OF HYDROGEN OXIDIZING BACTERIA.}

In order to obtain enrichment cultures we proceeded as follows. In an open Petri-dish of $13 \mathrm{~cm}$ diameter $50 \mathrm{cc}$ of an inorganic salt solution as used by GroHMANN and containing pro litre distilled water:

$\begin{array}{rll}1 & \text { gram } & \mathrm{Na}_{2} \mathrm{CO}_{3} \\ 1 & \text { " } & \mathrm{NH}_{4} \mathrm{Cl} \\ 0.7 & \text { " } & \mathrm{KH}_{2} \mathrm{PO}_{4} \\ 0.2 & \text { " } & \mathrm{MgSO}_{4} \\ 0.2 & \text { " } & \mathrm{NaCl} \\ \text { trace } & & \mathrm{FeCl}_{3}\end{array}$


was brought. After inoculation with some mud from a ditch the dish was placed at $30^{\circ} \mathrm{C}$. under a glass bell, in which the greater part of the air had been replaced by hydrogen and to which $10 \%$ carbon dioxide had been added. In a second experiment the procedure was the same, but this time the medium was inoculated with garden soil. In both experiments there was a marked gas consumption, and after 5 to 7 days a rather thick bacterial film had developed at the surface of the medium. These films were streaked off in the usual way on agar plates having the same mineral composition, which plates were brought in an anaerobic jar containing the same gas mixture as previously used. By replating four different pure cultures were obtained which were labeled Nr. 1 to 4 .

Preliminary experiments with these strains either in Eldredge tubes or in an apparatus as devised by SöHNGEN (11) showed that they all were able to oxidize hydrogen under autotrophic conditions.

All strains proved to be facultative heterotrophonts, growth on ordinary peptone agar being quite satisfactory.

For manometric experiments with resting bacteria strain $\mathrm{Nr} .2$ proved to be the most suitable, since the bacteria when grown autotrophically could readily be collected by centrifugation and from the sediment a homogeneous suspension could be obtained.

A further investigation of strain Nr. 2 showed that it was a Gramnegative motile, non sporeforming rod with lophotrichous cilia. On peptone agar with or without $1 \%$ glucose yellow colonies were formed. Gelatin was not liquefied, fermentative ability was lacking, in litmus milk no acid was formed, the litmus being reduced after some days. Neither indole nor hydrogen sulphide were produced.

These properties are in agreement with the idea that this culture was identical with Hydrogenomonas flava of NiKLEWSKI.

\section{EXPERIMENTS WITH RESTING BACTERIA.}

The first experiments with suspensions of Hydrogenomonas flava in the Warburg apparatus were most disappointing. In order to obtain without difficulty sufficiently dense suspensions, the bacterium had been grown under heterotrophic conditions. To our surprise these suspensions when brought in the Warburg apparatus into contact with gas mixtures containing hydrogen and oxygen in various proportions did only show a weak gas consumption identical with that in air, so that evidently no hydrogen had been oxidized.

This negative result induced us to study the behaviour of bacteria grown under strictly autotrophic conditions. With these bacteria indeed 
a strong gas consumption occurred in an-atmosphere consisting of a mixture of $80 \%$ hydrogen and $20 \%$ oxygen. However, the rate of gas consumption showed a marked decline with time to such a degree that after 30 to 90 minutes the consumption came to a standstill. Apparently the bacteria had died, since addition of an organic respiration substrate like lactate did not restore the gas consuming power.

The fact that NikLEwSKI reports that for hydrogen oxidation by his Hydrogenomonas flava it was necessary to reduce the oxygen content of the gas mixture considerably led to the establishment that a constant rate of gas consumption was only obtained when the amount of oxygen in the gas mixture was reduced to $8 \%$. This result tends to confirm our identification of strain Nr. 2 with the species in question.

In a first experiment the gas consumption of a bacterial suspension in $0.15 \mathrm{~mol}$ phosphate buffer $\mathrm{pH}=7.2$ in contact with a gas mixture containing $50 \% \mathrm{~N}_{2}, 42 \% \mathrm{H}_{2}$ and $8 \% \mathrm{O}_{2}$ was compared with the oxygen consumption of the same suspension in air both in the absence and in the presence of an organic respiration substrate. As such an aqueous solution of sodium lactate leading to a final concentration of $1 \%$ in the medium was added from a side-bulb. In all vessels a small quantity of $10 \%$ sodium hydroxide was present in the central compartment in order to absorb the carbon dioxide formed.

If in the first vessel hydrogen would act merely as a respiration substrate, one might expect that the amount of oxygen consumed would be the same as that consumed in the presence of the lactate. Since in the first case the consumption of one volume of oxygen would involve the disappearance of two volumes of hydrogen the total gas consumption should amount to thrice that in the lactate vessel.

For the sake of brevity we will refrain from giving here full particulars regarding this experiment. It may suffice to state that the gas consumption in the "hydrogen vessel" was at least 6 times that in the "lactate vessel" and from $40-50$ times as high as the oxygen consumption in the vessel to which no respiration substrate had been added.

This result might still be interpreted satisfactorily by assuming that the capacity of the dehydrogenase system in the bacteria which probably determines the rate of oxygen consumption is larger when molecular hydrogen replaces the organic hydrogen donator.

To test this possibility it was indicated to compare the gas consumption in the simultaneous presence of hydrogen and lactate with that in vessels to which only one of these oxidation substrates was added. This was done in the following experiment. 
Four manometric vessels all received $2 \mathrm{cc}$ of a bacterial suspension in $0.15 \mathrm{~mol}$ phosphate buffer $\mathrm{pH}=7.2$, whilst the central vessels all contained sodium hydroxide for the absorption of carbon dioxide formed.

For the rest the conditions were as follows:

$\mathrm{Vesse} 1 \mathrm{~A}$ : the side bulb contained $0.3 \mathrm{cc}$ distilled water

Vessel B: ", " , $\quad 0.3 \mathrm{cc}$ of a $6 \%$ solution of sodium lactate.

In both vessels $A$ and $B$ air was used as gas atmosphere.

Vessel C: as Vessel A

$\mathrm{Ves}$ e $1 \mathrm{D}$ : as Vessel $\mathrm{B}$

In both vessels $C$ and $D$ the gas atmosphere consisted of a mixture of $50 \% \mathrm{~N}_{2}, 42 \% \mathrm{H}_{2}$ and $8 \% \mathrm{O}_{2}$.

The results of this experiment are represented in Fig. 1.

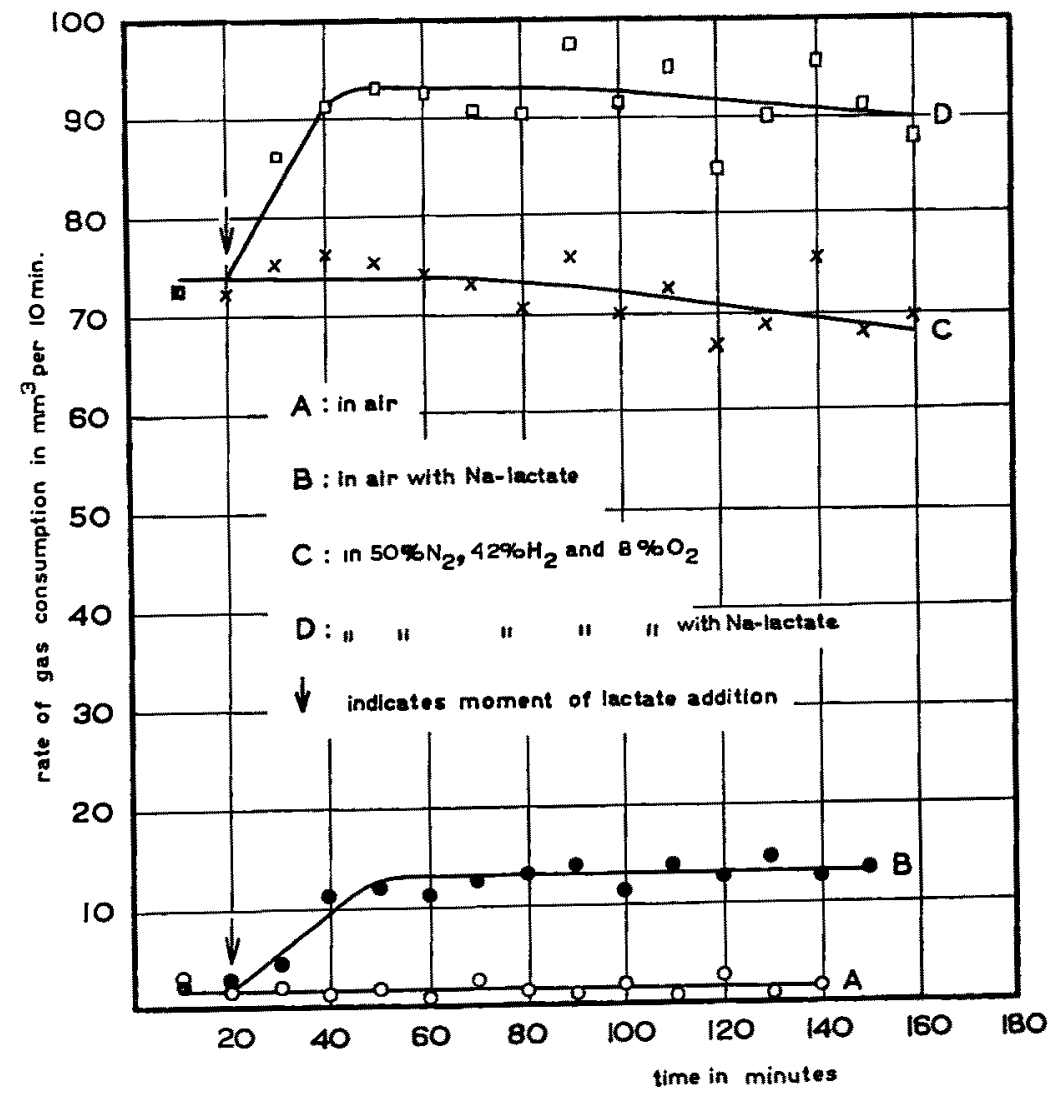

Fig. 1. Gas consumption by Hydrogemonas flava in air and in a hydrogenoxygen mixture with and without Na-lactate. 
In this figure the arrows indicate the moment at which the contents of the side bulb were added to the main vessel.

The outcome of the experiment is remarkable from several points of view. In the first place the results are in marked contrast to the current opinion, based mainly on the experiments of NikaEwSKI and of RuHLAND, that the presence of organic substrates prevents the oxidation of the hydrogen.

To the contrary comparison of the curves $C$ and D shows clearly that the gas consumption in the hydrogen containing atmosphere is also considerably increased by the addition of lactate.

The experiment may be considered a first proof that indeed hydrogen oxidation and lactate oxidation are independent processes as has been postulated by RUHLAND. It seems not well possible to reconcile this observation with the idea that both molecular hydrogen and lactate act as substrates for one and the same respiratory catalytic system. The protective action of organic substrates on the hydrogen oxidation as observed by earlier investigators must apparently be intimately linked up with the growth process.

A second point to which attention is drawn, is, however, that the effect of lactate addition to the hydrogen oxidizing system leads to an increase in rate of gas consumption which is almost twice as large as the rate of oxygen consumption in air. This means that the two gas consuming processes not only do not mutually interfere, but that an unmistakable stimulation of at least one of these processes takes place.

In order to obtain some further insight into what was going on under these conditions we decided to make some experiments in which the amount of lactate was taken so small that in case of oxidation it would be completely consumed within the duration of the experiment. If the effect of lactate addition would depend merely on a stimulation of the hydrogen oxidation one should expect that the gas consumption would remain constant at the high level. If on the other hand the lactate is oxidized simultaneously with the hydrogen the disappearance of the lactate out of the medium should also in the hydrogen containing atmosphere manifest itself in a return to the initial rate of gas consumption.

The results of such an experiment are collected in Fig. 2.

In this experiment 8 Warburg vessels were employed, four of which were filled with air, whilst in four others again a mixture of $50 \% \mathrm{~N}_{2}$, $42 \% \mathrm{H}_{2}$ and $8 \% \mathrm{O}_{2}$ was applied.

In each series the side bulb of one of the vessels contained $0.3 \mathrm{cc}$ distilled water, and the other three the same quantity of a solution of 


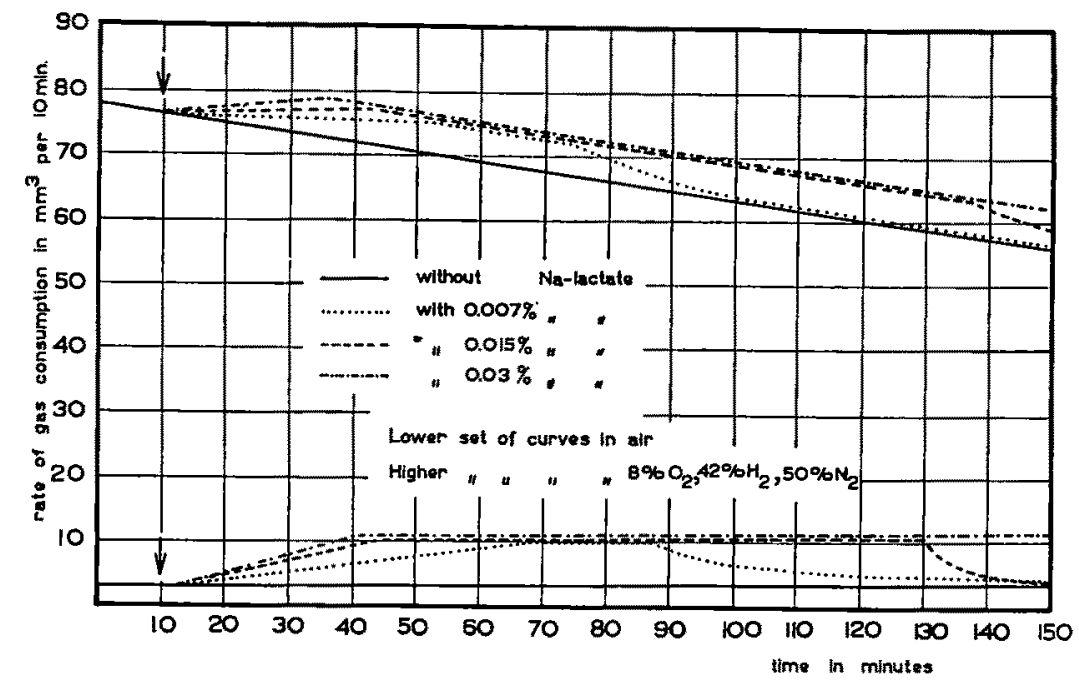

Fig. 2. The effect of the addition of different amounts of Na-lactate on the gas consumption by Hydrogenomonas flava in air and in a hydrogenoxygen mixture.

$\mathrm{Na}$-lactate in concentrations of $0.05 \%, 0.1 \%$ and $0.2 \%$ respectively This meant that after mixing the lactate concentrations in the media. became $0.007 \%, 0.015 \%$ and $0.03 \%$ respectively.

The outcome of the experiment is self-explanatory. From Fig. 2 it is obvious that the increase of gas consumption by the lactate addition to the medium in the hydrogen containing atmosphere does not only give rise to an increase in gas consumption which is almost equal to the increase in respiration in the air atmosphere, but that this rise in gas consumption extends over the same periods in both cases. This can only mean that also in the hydrogen containing atmosphere the lactate is oxidized at practically the same rate as in the normal respiration experiment. In this case we meet with a more or less ideal superposition of both gas consuming processes, which evidently proceed quite independently. This implies that in Hydrogenomonas flava a catalytic system for hydrogen oxidation is present which is quite apart from the catalytic systems involved in the normal respiration process.

We have corroborated these results by determining the so-called ,Knallgasquotient", i.e., the ratio of hydrogen and oxygen consumption, both in the absence and in the presence of lactate. Since the separate determination of the amounts of the two gasses disappearing in the vessels presents great difficulties we proceeded as follows. The vessels were first filled with hydrogen and hereupon an amount of oxygen was 
added which was kept so small that it was totally consumed before the end of the experiment.

After several trials the following technique was developed. Oxygen trom a bomb was led through an overflow in which a few centimeters of water pressure was maintained. With the aid of a two-way stopcock part of the oxygen at the reduced pressure could be led into a perforated stopper of the side-bulb of the Warburg-cuvette.

Before entering the cuvette the gas had been brought at the correct temperature by passing the gas through a glass spiral which was immersed into the waterbath. A correction for the fact that the capillary of the stopper contained air which was also pressed in could easily be made after the volume of the capillary had been determined.

The amount of oxygen pressed in could be accurately determined by reading the manometer which in these experiments was not filled with the usual Brodie solution, but with a solution of lead perchlorate having double the specific weight, as recommended by KREBS (12). For the preparation of this solution perchloric acid (s.w. 1.5) was neutralized with lead carbonate, after which $0.1 \%$ sodium cholate was added. After filtering off the precipitate the clear liquid was diluted with distilled water till the specific weight at room temperature was 2.068 .

The use of this manometer liquid means that the vessel constants had to be doubled. Taking this into account, it was quite feasible to introduce in each Warburg vessel equal volumes of oxygen.

Preliminary experiments showed that at the moment of total consumption of oxygen the gaseous metabolism practically came to a stand. Since the initial volume of the oxygen was known, is was possible to determine the volume of the hydrogen consumed at that moment from the total amount of gas which had disappeared.

The results obtained in two different experiments were as follows.

Ratio hydrogen/oxygen.

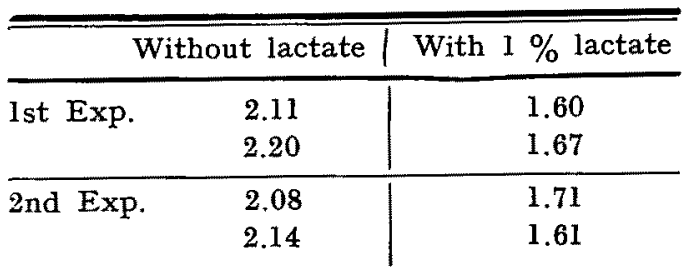

From these figures too it is evident that addition of lactate does not lead to a mere stimulation of the hydrogen oxidation, i.e., an increased 
consumption of both hydrogen and oxygen, but that this addition specifically increases the oxygen consumption, which can only mean that next to the hydrogen oxidation an oxidation of the lactate also takes place.

The second point which we have investigated is the question whether Hydrogenomonas flava is able to promote a reaction between hydrogen and carbon dioxide as postulated by KASERER and others. In order to study this question the autotrophically grown bacteria have been suspended in an bicarbonate buffer and the suspension brought into contact with hydrogen. Although we have repeated this experiment several times, in no case any decrease in pressure could be observed. In all cases we have convinced ourselves that the bacteria were in an active state by admitting after some time a small quantity of oxygen. This always led to an immediate gas consumption.

\section{DISCUSSION OF RESULTS.}

Although this investigation bears only a preliminary character, a few conclusions seem to be warranted.

For a typical hydrogen oxidizing bacterium, in all probability identical with Hydrogenomonas flava Niklewski, it has been shown that it is possible to superpose a normal respiration on the process of hydrogen oxidation. This result is contrary to what might be expected on ground of various statements in literature, according to which the addition of organic respiration substrates to cultures of hydrogen oxidizing bacteria practically completely inhibits the hydrogen oxidation. Apparently the explanation of this contradiction must be sought in the fact that the addition of organic substrates to the cultures leads to the generation of bacteria of a purely heterotrophic character, lacking the faculty to bring about hydrogen oxidation. Our experiments make it probable that hydrogen oxidation asks for the presence of a special catalytic system which is quite independent of the catalytic system active in the normal respiration. Obviously the formation of the special hydrogen oxidizing system only takes place when the bacteria are grown under autotrophic conditions, a statement which is borne out by our observation that suspensions of Hydrogenomonas flava grown under heterotrophic conditions are unable to bring about any hydrogen oxidation when tested as resting bacteria in the Warburg apparatus.

Yet it should be kept in mind that the faculty to produce the catalytic system in question is only gradually lost. Throughout our investigation we have relied on pure cultures of Hydrogenomonas flava grown hetero- 
trophically. Nevertheless, it remained possible to transfer these cultures successfully in media suitable for autotrophic development, and it were the crops obtained in these autotrophic cultures which have been used in the manometric experiments. However, at the end of our investigation we have been obliged to witness that the autotrophic cultures no longer succeeded; apparently the faculty to build up the special hydrogen oxidizing apparatus had irreparably gone lost by the continued cultivation of the strain under heterotrophic conditions. We are the more inclined to accept this explanation with a view to similar experiences of earlier investigators, in which connection we refer to the paper of GroHMANN (8).

For the problem of chemo-autotrophic carbon dioxide assimilation our results may be deemed of importance, in so far as it has been shown that this assinilation does not take place in the absence of oxygen. Herewith the theory first advanced by KASERER, supported by NikLEwSKI and more recently by BURK, according to which the primary reaction is one between carbon dioxide and hydrogen, may be deemed to be definitely refuted. Evidently carbon dioxide assimilation can only proceed with simultaneous consumption of oxygen, and for the moment we must conclude that in analogy with what is known regarding the chemoautotrophy in other bacterial groups the hydrogen oxidation is in some way or other indispensable for the carbon dioxide assimilation. This means, of course, that the riddle of the mechanism of this assimilation remains unsolved.

\section{Sum mary.}

Enrichment cultures for hydrogen oxidizing bacteria enabled us to isolate four different species having this faculty, one of which apparently was identical with Hydrogenomonas flava NikJewski. This strain proved to be suitable for manometric experiments with resting bacteria.

With suspensions of these bacteria manometric experiments regarding the gaseous metabolism have been performed with the ultimate aim of contributing to the solution of the problem of chemo-autotroplic carbon dioxide assimilation.

It was found that the bacteria only brought about hydrogen oxidation, if they had also been grown under autotrophic conditions; heterotrophically grown bacteria did not oxidize molecular hydrogen, although they were able to respire normally with organic substrates.

In contrast herewith the autotrophically grown bacteria proved to 
be able to bring about both types of oxidation. Moreover, on addition of both hydrogen and lactate these substrates proved to be oxidized simultaneously, the rate of each of these oxidations being unimpaired. Under certain conditions the total gas consumption was even unmistakebly enhanced. These results seem to warrant the conclusion that hydrogen oxidation asks for a special catalytic system independent of the catalysts active in the normal respiration process. Apparently this special system is only built up when the bacteria are grown under autotrophic conditions, and the faculty to synthesize this system is gradually - and after some time irreparably - lost on cultivation under heterotrophic conditions.

Finally it has been shown that bacteria, which were quite active as far as hydrogen oxidation was concerned, were completely unable to bring about a reaction between hydrogen and carbon dioxide. Herewith the theory regarding the nature of the metabolism of hydrogen oxidizing bacteria already advanced in 1906 by KASERER and still recently defended by BuRK has been definitely refuted.

\section{References.}

1. Th. De Saussure, Mem. de la Soc. d. phys. et d'hist. nat. de Genève 8. 163, 1839. - 2. H. IMMENDORF, Landw. Jahrb. 21, 281, 1892. - 3. B. NikLewski, Extr. du Bull. de l'Acad. des sciences de Cracovie, 911, 1906. 4. H. KASERER, Zeitschr. f. d. landw. Versuchswesen in Oesterreich 8, 789, 1905; Zentralbl. f. Bakt. II, 16, 681 u. 769, 1906. - 5. A. F. LEBEDEFF, Biochem. Zeitschr. 7, 1, 1908; Ber. d. deutsch. bot. Ges. 27, 598, 1909. 6. B. NIKLEWSKI, Jahrb. f. wissensch. Bot. 48, 111, 1910. - 7. W. RuHLAND, Jahrb. f. wissensch. Bot. 63, 321, 1924. - 8. H. Grohmann, Zentralbl. f. Bakt. II, 61, 256, 1924: - 9. S. B. LeE and W. W. UMBREIt, Zentralbl. f. Bakt. II, 101, 354, 1940. - 10. D. BURk, Journ. Phys. Chem. 35, 432, 1931. - 11. N. L. SöHngen, Het ontstaan en verdwijnen van waterstof en methaan onder den invloed van het organische leven. Delft, 1906. - 12. H. A. KREBS, Biochem. Zeitschr. 220, 250, 1930. 